\title{
Mountable Scattered Facilitating Reliability Evidence for Software-as-a-Service Clouds
}

\author{
M. Nanda Kishore \\ Assistant Professor \\ SVCET, Chittoor (AP)
}

\author{
S. Palani \\ Assistant Professor \\ SVCET, Chittoor (AP)
}

\author{
T. Vanitha \\ PG Scholar \\ SVCET, Chittoor (AP)
}

\begin{abstract}
Programming as-an administration (SaaS) cloud frameworks empower application administration suppliers to convey their applications through huge distributed computing foundations. Not with standing, because of their imparting nature, SaaS mists are powerless against noxious assaults. In this paper, we show IntTest, an adaptable and viable administration honesty verification system for SaaS mists. IntTest gives a novel coordinated authentication chart investigation conspire that can give stronger aggressor pinpointing force than past plans. Additionally, IntTest can naturally upgrade result quality by supplanting awful results created by noxious aggressors with great results delivered by considerate administration suppliers. We have actualized a model of the IntTest framework and tried it on a creation distributed computing foundation utilizing IBM System $S$ stream preparing applications. Our trial results demonstrate that IntTest can accomplish higher aggressor pinpointing exactness than existing methodologies. IntTest does not require any exceptional equipment or secure bit bolster and forces little execution effect to the application, which makes it pragmatic for extensive scale cloud frameworks.
\end{abstract}

\section{General Terms}

Scattered Facility Reliability Attestation, Cloud Computing, Protected Scattered Data Handling.

\section{Keywords}

SaaS, SOA, ASPs.

\section{INTRODUCTION}

CLOUD registering has developed as a savvy asset renting standard, which bviates the requirement for clients keep up complex physical processing infrastructures without anyone else. Programming as-an administration (SaaS) mists expand upon the ideas of programming as an administration and administration situated structural planning (SOA), which empower application administration suppliers (ASPs) to convey their applications through the monstrous distributed computing base. Specifically, our work concentrates on information stream transforming administrations that are thought to be one class of executioner applications for mists with numerous genuine applications in security reconnaissance, logical registering, and business knowledge. Notwithstanding, distributed computing frameworks are regularly imparted by ASPs from distinctive security areas, which make them helpless against malicious as saults . Case in point, assailants can put on a show to be real administration suppliers to give fake administration segments, and the administration parts gave by considerate administration suppliers may incorporate security gaps that can be misused by aggressors. IntTest gives a handy administration honesty confirmation plot that does not accept trusted elements on outsider administration provisioning destinations or oblige application alterations. IntTest expands upon our past work RunTest and AdapTest yet can give stronger pernicious assailant pinpointing force than RunTest and AdapTest. In particular, both RunTest and AdapTest and also conventional lion's share voting plans need to expect that benevolent administration suppliers take larger part in every administration capacity. On the other hand, in expansive scale multitenant cloud systems, numerous noxious aggressors may dispatch plotting assaults on certain focused on administration capacities to negate the supposition. To address the test, IntTest takes an all encompassing approach by deliberately analyzing both consistency and irregularity connections among distinctive administration suppliers inside the whole cloud framework.

Which empower application administration suppliers (ASPs) to convey their applications through the huge distributed computing base. Specifically, our work concentrates on information stream handling administrations that are thought to be one class of executioner applications for mists with numerous certifiable applications in security reconnaissance, investigative registering, and business brainpower. Then again, distributed computing foundations are regularly imparted by ASPs from diverse security spaces, which make them helpless against malevolent assaults. Case in point, aggressors can profess to be genuine administration suppliers to give fake administration parts, and the administration segments gave by considerate administration suppliers may incorporate security gaps that can be misused by assailants. The disadvantages are,

- Those strategies frequently oblige extraordinary trusted equipment or secure portion support.

- Which makes them hard to be sent on extensive scale distributed computing foundations.

We exhibit IntTest, another coordinated administration honesty validation structure for multitenant cloud frameworks. IntTest gives a handy administration uprightness confirmation plot that does not expect trusted elements on outsider administration provisioning locales or oblige application alterations. IntTest expands upon our past work Run Test and AdapTest yet can give stronger pernicious assailant pinpointing force than Run Test and AdapTest. In particular, both RunTest and AdapTest and additionally conventional larger part voting plans need to accept that amiable administration suppliers take lion's share in every administration capacity. Notwithstanding, in vast scale multitenant cloud frameworks, different malignant assailants may dispatch plotting assaults on certain focused on administration capacities to discredit the presumption. To address the test, IntTest takes an all encompassing approach by deliberately looking at both consistency and irregularity 
connections among distinctive administration suppliers inside the whole cloud framework. IntTest looks at both every capacity consistency diagrams and the worldwide.

\section{SYSTEM ARCHITECTURE}

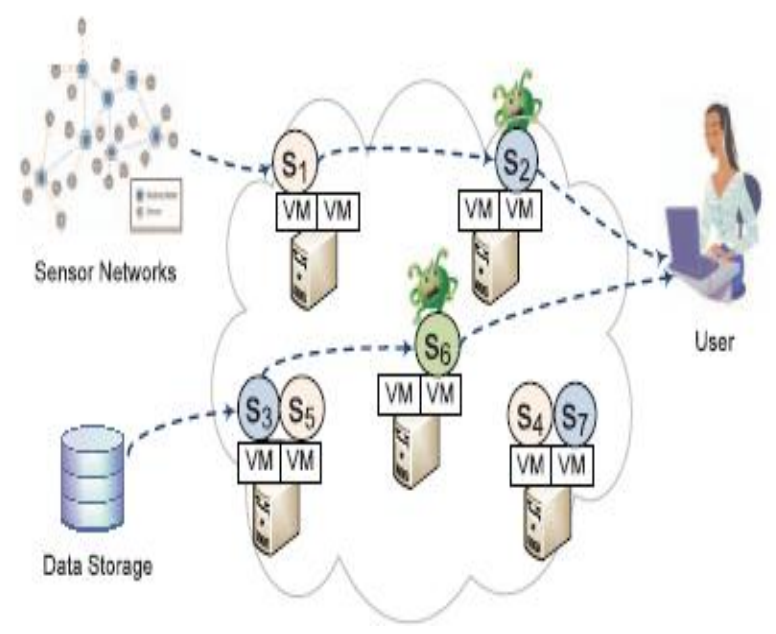

Figure: 2.1 System Architecture Diagram

Usually Data Users can extract information from everywhere. In this project, user can get data from Sensor networks and Data storage through servers. Due to Lack of security, attackers can interrupt the flow of data.

\subsection{Baseline Attestation}

Our methodology is that if two administration suppliers can't help contradicting one another on the handling aftereffect of the same information, no less than one of them ought to be vindictive. Note that we don't send a data information thing and its copies simultaneously. Rather, were play the validation information on diverse administration suppliers in the wake of getting the preparing consequence of the first information. Hence, the malevolent aggressors can't stay away from the danger of being distinguished when they deliver false results on the first information. In spite of the fact that the replay plan may bring about postponement in single tuple preparing, we can Overlap the authentication and ordinary handling of sequential tuples in the information stream to conceal the confirmation delay from the client. In the event that two administration suppliers dependably give steady yield comes about on all info information, there exists consistency connection ship between them. Something else, on the off chance that they give diverse yields on no less than one info information, there is irregularity relationship between them. We don't restrict the consistency relationship to balance capacity since two amiable administrations suppliers may create comparable however not precisely the same results.

\subsection{Integrated Attestation}

\subsubsection{Consistency chart examination}

We first analyze every capacity consistency diagrams to pinpoint suspicious administration suppliers. The consistency interfaces in every capacity consistency charts can tell which set of administration suppliers keep steady with one another on a particular administration capacity.Given any administration capacity, since considerate administration suppliers dependably keep steady with one another, amiable administration suppliers will frame a coterie regarding consistency links. However, deliberately conniving assailants can attempt to take greater part in a particular administration capacity to escape the identification. In this way, it is deficient to inspect the every capacity consistency diagram just. We have to incorporate the consistency chart examination with the irregularity diagram analysis to accomplish more powerful respectability authentication.

\subsubsection{Inconsistency diagram examination}

Given an irregularity chart containing just the irregularity joins, there may exist diverse conceivable mixes of the kindhearted hub set and the pernicious hub set. In any case, in the event that we accept that the aggregate number of noxious administration suppliers in the entire framework is close to $\mathrm{K}$, we can pinpoint a subset of really vindictive administration suppliers. Instinctively, given two administration suppliers joined by an irregularity join, we can say that no less than one of them is malevolent since any two generous administration suppliers ought to dependably concur with one another. In this way, we can determine the lower bound about the quantity of vindictive administration suppliers by inspecting the base vertex front of the irregularity diagram. The base vertex front of a diagram is a base situated of vertices such that every edge of the chart is occurrence to atleast one vertex in the set.

\section{AUTO CORRECTION FOR ATTACKS}

IntTest can pinpoint vindictive administration suppliers as well as naturally rectify adulterated information handling results to enhance the outcome nature of the cloud information preparing administration, without our verification plan, once a unique information thing is controlled by any malicious hub, the transforming aftereffect of this data thing can be undermined, which will bring about degraded result quality. IntTest influences the confirmation information and them malicious hub pinpointing results to distinguish and correct compromised information handling results.

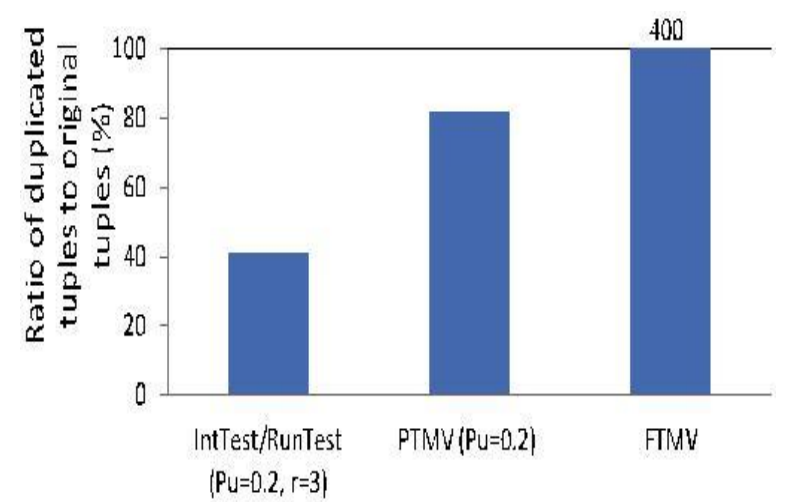

Figure: 3.1 Auto Correction for Attacks Ratio

An adaptable and proficient conveyed administration respectability verification system for large scale distributed computing bases. A novel incorporated administration honesty verification conspire that can attain to higher pinpointing precision than past strategies. An outcome autocorrection method that can consequently remedy the impure results delivered by malicious attackers. Both scientific study and exploratory assessment to measure the precision and overhead of the incorporated administration uprightness authentication plan. 


\section{RESULT AND DISCUSSIONS}

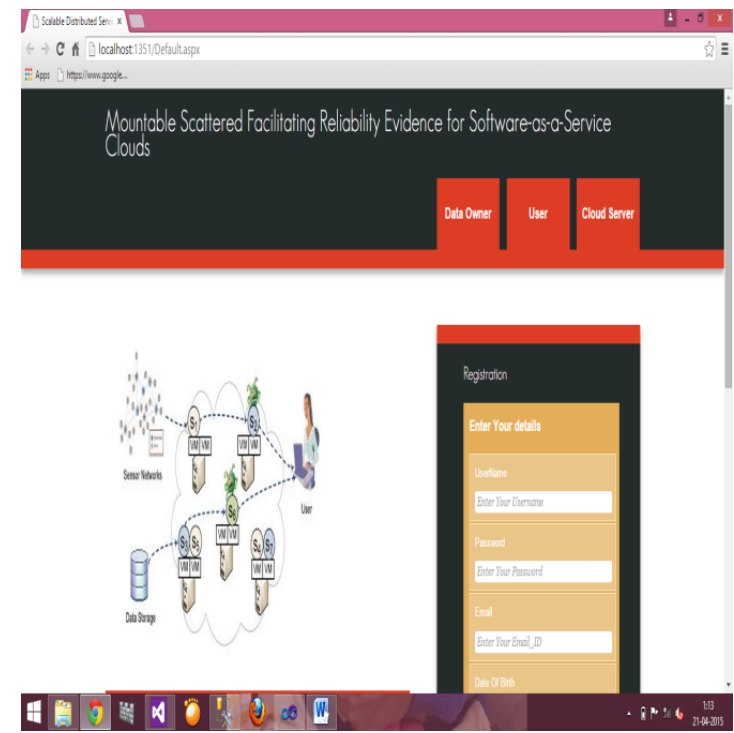

Figure 4.1: Registration Page

This Registration Page is used to register the details of owner and user .After entering the details correctly, it displays the message as Registered successfully.

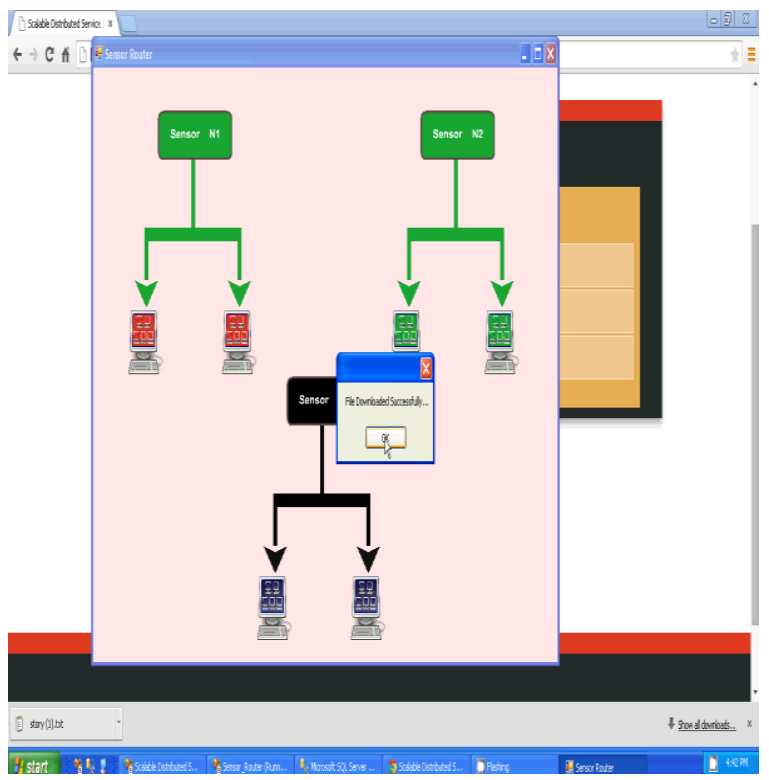

Figure: 4.2 Sensor Router
While uploading a File, we must choose a file from the folder then choose the server in which server you need to upload a file. Before uploading a file the sensors will be displayed in Black color. After uploading a file, it will be changed to Green color and it displays a message file uploaded successfully.

\section{CONCLUSION}

We have displayed the outline and implementation of IntTest, a novel coordinated administration uprightness validation structure for multitenant programming as-an administration cloud frameworks. IntTest utilizes randomized replay-based consistency check to confirm the honesty of circulated administration parts without forcing high overhead to the cloud framework. IntTest performs coordinated examination over both consistency and irregularity verification charts to pinpoint conniving assailants all the more effectively than existing methods. Moreover, IntTest gives result autocorrection to consequently redress traded off results to enhance the outcome quality. We have executed IntTest and tried it on a business information stream processing stage running inside a generation virtualized distributed computing foundation. Our exploratory results demonstrate that IntTest can attain to higher pinpointing exactness than existing option plans. IntTest is lightweight, which forces low-execution effect to the information handling administrations running inside the distributed computing framework.

\section{ACKNOWLEDGEMENT}

We thank our project guide Mr.M.Nanda kishore \& S. Palani for their valuable guidance and for providing all the necessary facilities, which were indispensable in completion of this paper for helpful discussions and comments.

\section{REFERENCES}

[1] Amazon web Services http://aws.amazon.com/, 2013.

[2] Google App Engine, http://code.google.com/appengine/, 2013.

[3] G. Alonso, F. Casati, H. Kuno, and V. Machiraju, Web Services Concepts, Architectures and Applications (Data-Centric Systems and Applications). AddisonWesley Professional, 2002.

[4] T. Erl, Service-Oriented Architecture (SOA): Concepts, Technology, and Design. Prentice Hall, 2005.

[5] T.S. Group, "STREAM: The Stanford Stream Data Manager," IEEE Data Eng. Bull., vol. 26, no. 1, pp. 1926, Mar. 2003.

[6] D.J. Abadi et al., "The Design of the Borealis Stream Processing Engine," Proc. Second Biennial Conf. Innovative Data Systems Research (CIDR '05), 2005. 